\title{
In Situ Studies for Understanding Intragranular Nanopore Evolution in Ni-rich Layered Oxide Cathode Material
}

\author{
A.Pokle $^{1 *}$, S. Ahmed ${ }^{1}$, A. Beyer ${ }^{1}$, S. Schweidler ${ }^{2}$, M. Bianchini ${ }^{2}$, P. Hartmann ${ }^{2}$, T. Brezesinski ${ }^{2}$, J. \\ Janek $^{2,3}$ and K. Volz ${ }^{1}$ \\ ${ }^{1}$ Materials Science Center and Faculty of Physics (WZMW), Philipps University Marburg, Germany \\ ${ }^{2}$ KIT/BASF Battery and Electrochemistry Laboratory (BELLA), Institute of Nanotechnology, Karlsruhe \\ Institute of Technology (KIT), Hermann-von-Helmholtz-Platz 1, 76344 Eggenstein-Leopoldshafen, \\ Germany \\ ${ }^{3}$ Physikalisch-Chemisches Institut, Justus-Liebig-Universität Gießen, Germany \\ * Corresponding author: pokle @ staff.uni-marburg.de
}

Understanding the electrode material behavior under various conditions is critical for developing nextgeneration battery technologies. Dynamic conditions inside the working battery cell have been recently explored using advanced in-situ characterization techniques. Despite widespread applications and research investments, energy storage materials still face a lack of complete understanding. Performing real-time electron microscopy is one of the significant developments, which has enabled us to probe local chemo-mechanical changes under dynamic conditions.

The requirement for higher energy densities within lithium ion batteries lead to the development of Nirich NCMs. Within this class we investigated a material with $85 \% \mathrm{Ni}$ : $\mathrm{Li}_{1+\mathrm{x}}\left(\mathrm{Ni}_{0.85} \mathrm{Co}_{0.10} \mathrm{Mn}_{0.05}\right)_{1-\mathrm{x}} \mathrm{O}_{2}$. Typically, NCMs are synthesized as secondary particles made from densely packed primary particles. Strain is induced within the primary particles during charging/discharging cycles due to volume change, which can result in cracking of the secondary particles. Previously, surface reconstruction from a layered structure to a rock salt structure (refer figure 1), the formation of inter/intragranular cracks, and pores (or voids) have been reported. Among these, the pore formation in cycled materials is not well characterized, and its presence in pristine samples is usually neglected [1], [2]. Pore formation has been attributed to the release of oxygen in work carried out by Linqin $\mathrm{Mu}$ et al. [3].

Here, we report the origin of nanopores and investigate the heating and biasing effects using in-situ heating/biasing holder. Intragranular nanopores are observed in a wide variety of layered oxide cathode materials, but so far it is not clear if and how they affect the performance of the materials. By employing Focussed Ion Beam, Aberration-Corrected STEM (AC-STEM) and Electron Energy Loss Spectroscopy with 4DSTEM capabilities, we study the effect of nanopores on the intragranular structure of the primary particles during cycling. In addition to observing phase transformation (leading to metal segregation) and inter/intragranular cracks, we have also investigated cation disordering within the nanopore at sub-angstrom resolution. 
References:

[1] P. Yan, J. Zheng, M. Gu, J. Xiao, J.-G. Zhang, and C.-M. Wang, "Intragranular cracking as a critical barrier for high-voltage usage of layer-structured cathode for lithium-ion batteries," Nat Commun, vol. 8, Jan. 2017.

[2] J. Zheng et al., "Suppressed oxygen evolution induced degradation at high charge cut-off voltages in LiNixMnyCozO2 cathodes," Nano Research, no. Tsinghua University Press and Springer-Verlag, p. 20. [3] L. Mu et al., "Oxygen Release Induced Chemomechanical Breakdown of Layered Cathode Materials," Nano Lett., vol. 18, no. 5, pp. 3241-3249, May 2018.

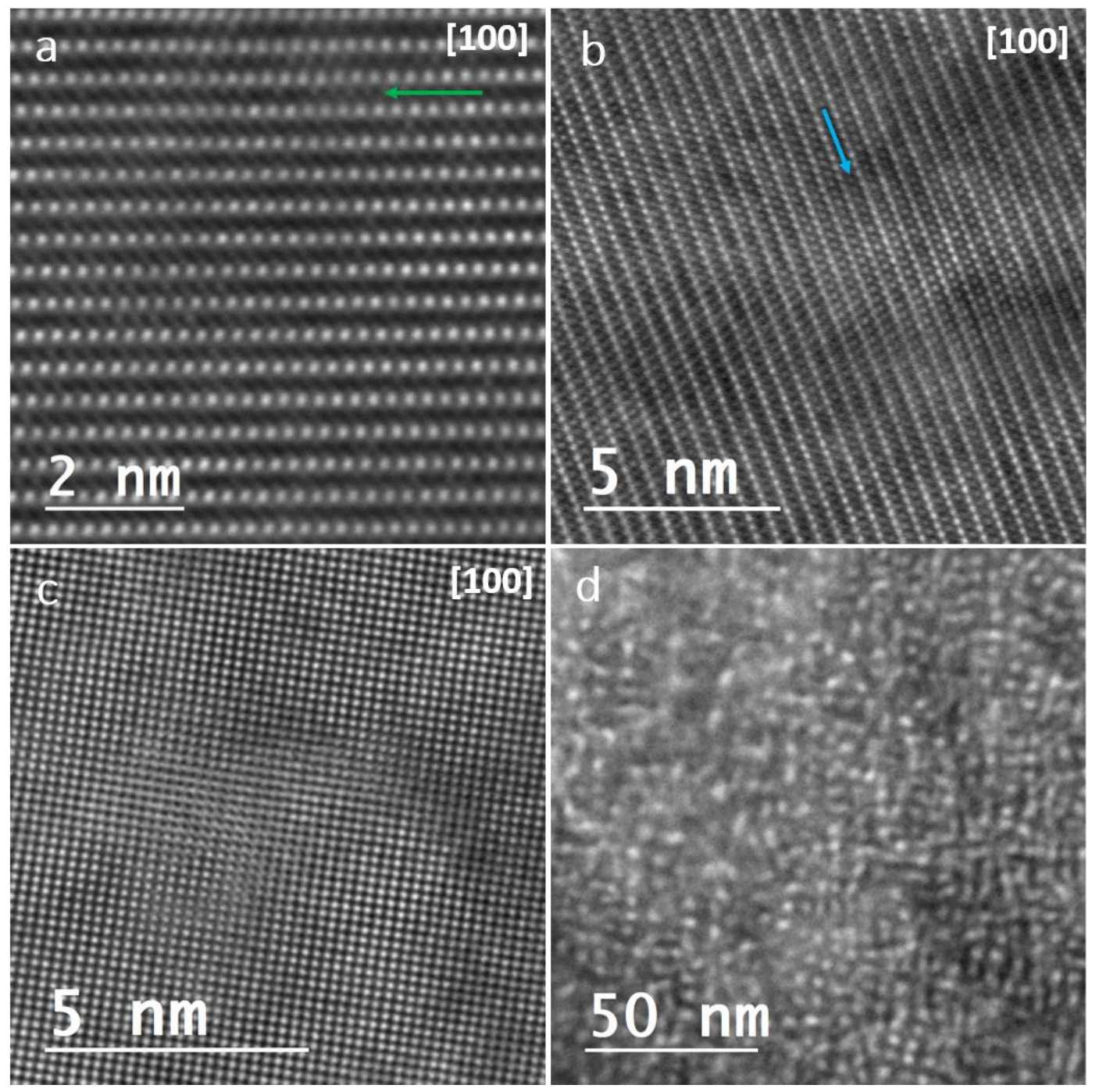

Figure 1: HAADF micrographs using AC-STEM of NCM851005. (a) showing layered structure, the green arrow marks the undetected Li position ( $\mathrm{O}$ atomic columns exhibiting diffused contrast) between the TM rows in uncycled sample, (b) 200-cycled displaying TM replacing Li positions (blue arrow exhibiting sharp contrast), (c) high-resolution image from region (d) showing rock salt (cubic) structure. (d) surface appearance at low magnification of the region that has been transformed into rock salt structure in 500-cycled sample. 